\title{
CMA members gathered in Quebec for 150th annual meeting
}

\author{
— Cite as: CMAJ 2017 September 5;189:E1125-6. doi: 10.1503/cmaj.1095478
}

Posted on cmajnews.com on Aug. 17, 2017, updated on Aug. 23, 2017.

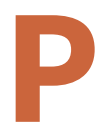

hysicians from across Canada met in Quebec to discuss the biggest issues and challenges in the medical profession. The 150th General Council and Annual Meeting of the Canadian Medical Association (CMA) was held in Quebec City on Aug. 20-23. The major themes of this year's gathering included physician innovation, medical professionalism and advocacy in medicine.

Other topics on the agenda included issues that continue to dominate health care news headlines, such as the ongoing opioid crisis and medically assisted dying. Several of the topics CMA members discussed are explained in more detail below.

\section{The opioid crisis}

There were at least 2458 "apparent opioid-related deaths" in Canada in 2016, a rate of 8.8 deaths per 100000 population, according to a federal government report. The data do not include statistics from Quebec. Overall, reporting on opioid-related deaths has been inconsistent.

It is "very difficult to address a problem that is not accurately measured," Dr. Jane Philpott, the federal health minister, has stated. Philpott has also noted that the death toll of the opioid crisis is "worse than any other infectious epidemic in Canada, including the peak of AIDS deaths."

The federal government has committed more than $\$ 100$ million to help efforts to address substance abuse. On Aug. 15, Prime Minister Justin Trudeau reiterated on Twitter that "We're doing everything we can to help fight this ongoing opioid crisis from every angle and keep Canadians safe."

British Columbia has been ground zero for the opioid epidemic in Canada. This

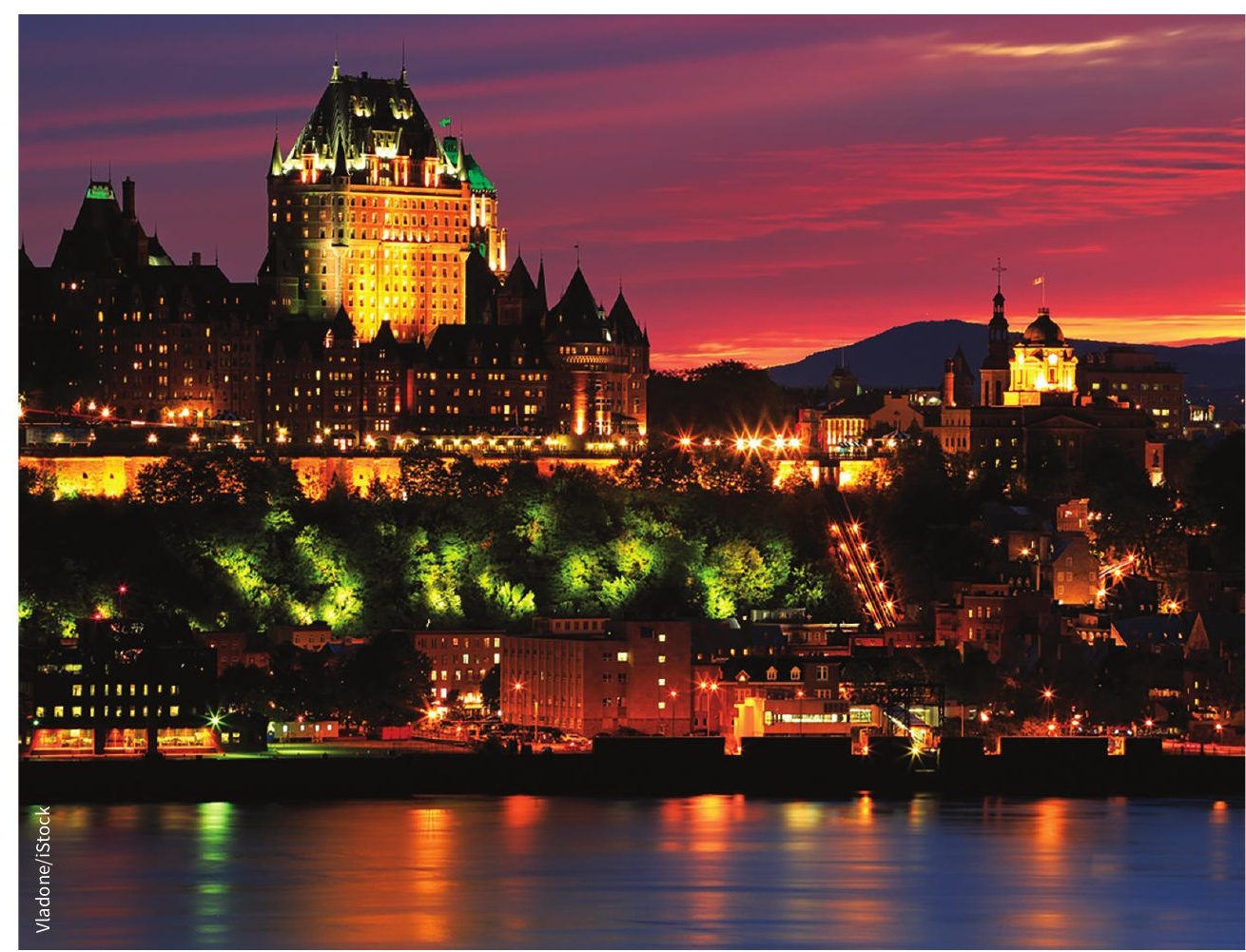

The 150th General Council and Annual Meeting of the Canadian Medical Association was held in Quebec City on Aug. 20-23.

year, up to May 31, there have already been 525 illicit drug overdose deaths involving fentanyl. That's a 109\% increase over the same period last year. Fentanyl was detected in $78 \%$ of overdose deaths involving illicit drugs this year.

Alberta has also released statistics for 2017, reporting 176 apparent overdose deaths related to fentanyl as of May 13. The opioid problem is also worsening in Ontario, which saw more than 700 opioidrelated deaths in 2015, a 99\% increase since 2003. The province has not released more recent statistics.

Other provinces, however, have experienced very few opioid-related overdose deaths. As of July 10 , Saskatchewan has reported only one opioid-related death. New Brunswick, Prince Edward Island and Nova Scotia have also reported five or fewer probable opioid-related deaths.

The CMA has noted that, although opioids still offer legitimate therapeutic benefits, the "prescribing of opioids must be done in a manner that is based on evidence, and includes careful assessment and monitoring." CMAJ published a new guideline on opioid therapy and chronic noncancer pain on May 8. At general council, CMA delegates focused on "how physician action and advocacy can help reduce the damage caused by opioids." 
Medical assistance in dying

At general council this year, delegates discussed assisted dying issues that have been identified as complex and in need of further study. Specifically, discussion focused on advance requests and requests from mature minors or people whose sole underlying condition is psychiatric.

The legislation on medical assistance in dying, Bill C-14, became law on June 17, 2016. The three types of complex requests mentioned above, however, remain under debate across the country. In December of 2016, the federal government asked the Council of Canadian Academies to "undertake independent reviews" to examine these issues. An expert panel was formed - comprising 44 members from disciplines such as law, medicine, ethics and the social sciences - and will be accepting input from groups and individuals affected by Bill C-14 until Sept. 22. The panel is expected to release its findings in late 2018.

Some groups have already publicly disclosed their positions. The Canadian Bar Association, which represents 36000 lawyers, supports amending the existing legislation to accommodate all three types of complex requests. "Additional study may offer value, particularly in developing patient-centric and appropriate tools to assess the capacity of persons with psychiatric conditions and children and youth," the association wrote in a letter to the federal ministers of health and justice.
The advocacy group Dying with Dignity Canada claims that Bill C-14 discriminates against certain groups of desperately ill Canadians and violates the Canadian Charter of Rights and Freedoms. It has expressed disappointment that the outcome of the work of the Council of Canadian Academies will only be reports and not specific policy recommendations. On the group's website, CEO Shanaaz Gokool took issue, in particular, with the lack of accommodation for advance requests in the existing law.

"Without the option to make an advance request for assisted dying while they still have capacity, Canadians with dementia, or other conditions that rob victims of their mental faculties, will continue to be denied their hard-won right to a peaceful death," stated Gokool.

Many CMA members, however, appear more wary of providing medically assisted dying to certain patient groups. In a survey of CMA members from June 2016, a quarter of respondents indicated they would participate in medically assisted deaths. Of those, only $22 \%$ would provide it when the sole diagnosis is mental illness, $38 \%$ would provide it for mature minors, and 59\% would provide it for nonterminal illnesses.

\section{Revision of CMA code of ethics}

According to the CMA, its code of ethics, which dates to 1868 , is "arguably the most important document" it produces. "It has a long and distinguished history of providing ethical guidance to Canada's physicians."

Topics included in the code of ethics include physicians' responsibilities to patients, to the profession, to society and to themselves. Among the responsibilities considered fundamental in the code are putting the patient's needs first, engaging in lifelong learning, resisting influence that could undermine integrity, and promoting physician well-being.

The current code was last updated in 2004. At general council, there were discussions on contemporary medical issues and emerging practices to help guide a "substantive revision" of the code. Principles of medical professionalism will also be incorporated into the code, which will be renamed to the CMA Code of Ethics and Professionalism.

\section{Other topics}

Delegates discussed physician innovation and explored the barriers to "developing a culture of innovation within the profession." One session focused on promoting civility within the profession, using CMA findings on intraprofessionalism to "assess and decipher key considerations and challenges that divide the medical profession today." Participants in a primer on physician advocacy learned how to better engage Members of Parliament on matters of health policy.

\section{Roger Collier, CMAJ}

